\title{
A Self Organization Approach to Goal-directed Multimodal Locomotion Based on Attractor Selection Mechanism
}

\author{
Yongjae Kim $^{1,2}$, Surya G. Nurzaman ${ }^{2,3,4}$, Fumiya Iida $^{2,3}$ and Edwardo F. Fukushima ${ }^{5}$
}

\begin{abstract}
The realization and utilization of multimodal locomotion to enable robots to accomplish useful tasks is a significantly challenging problem in robotics. Related to the challenge, it is crucial to notice that the locomotion dynamics of the robots is a result of interactions between a particular control structure and its body-environment dynamics. From this perspective, this paper presents a simple control structure known as Attractor Selection Mechanism that enables a robot to self organize its multiple locomotion modes for accomplishing a goal-directed locomotion task. Despite the simplicity, the approach enables the robot to automatically explore different body-environment dynamics and stabilize onto particular attractors which corresponds to locomotion modes relevant to accomplish the task. The robot used throughout the paper is a curved-beam hopping robot, which despite its simple actuation method, possesses rich and complex bodyenvironment dynamics.
\end{abstract}

\section{INTRODUCTION}

In the biologically inspired robotics field, multimodal locomotion ability, i.e. the ability to perform multiple distinguishable modes of locomotion by a single robot, has gained significant attractions due to its potential to enable robots accomplishing a variety of useful tasks. For example, a number of researches focused on the realization of different locomotion modes depending on the ecosystems, inspired by animals like salamanders and frogs [1,2]. On the other hand, many others also focused on multimodal locomotion in terrestrial environments such as walking and running by a single robot with a compliant legged system similarly possessed by humans [3].

However, in order to take advantage of multiple locomotion modes, it is important to notice that the dynamics of robots as embodied systems must be seen as a coupled dynamics among their particular control architectures, bodies and environments [4-6]. Therefore, it is crucial to use a

\footnotetext{
${ }^{1}$ Authors are with Department of Mechanical and Aerospace Engineering, Tokyo Institute of Technology, 2-12-1 I1-68, Ohokayama, Meguro-ku, Tokyo, Japan kim.y.ae@m.titech.ac.jp

${ }^{2}$ Authors are with Bio-Inspired Robotics Laboratory, Institute of Robotics and Intelligent Systems, Department of Mechanical and Process Engineering, ETH Zürich, Leonhardstrasse 27, 8092 Zurich, Switzerland surya.nurzaman@mavt.ethz.ch, fumiya.iida@mavt.ethz.ch

${ }^{3}$ Authors are with Machine Intelligence Laboratory, Department of Engineering, Cambridge University, Trumpington Street, Cambridge, United Kingdom sgn26@cam.ac.uk, fi224@cam.ac.uk

${ }^{4}$ Authors are with the Department of Mechanical Engineering, School of Engineering, Monash University, Sunway Campus, Jalan Lagoon Selatan, Bandar Sunway, Selangor, Malaysia surya.nurzaman@monash.edu

${ }^{5}$ Authors are with the Department of Mechanical Engineering, Tokyo University of Technology, 1404-1 Katakura, Hachiouji, Tokyo, Japan fukushimafmhkestf.teu.ac.jp
}

control architecture that can realize and utilize multiple locomotion modes in a self organizing fashion. This essential means that the control structure enables the robots to explore different body-environment dynamics and stabilize themselves onto particular attractors corresponding to locomotion modes relevant to the robots' task.

Inspired by neural mechanisms in animals, some studies have proposed the use of an adaptive chaotic search in robots as a control architecture to explore a variety of bodyenvironment dynamics, i.e. the resulting dynamics due to the interactions between the robot's body and the environment [7]. However, other works focus on simpler animals and suggest that their dynamics is driven by inherent stochasticity. For instance, in terms of goal-directed locomotion, it is suggested that chemotaxis in bacteria such as Escherichia coli and nematodes Caenorhabditis elegans is achieved through a random walk biased by sensory stimulation [8,9].

The main goal of this paper is to propose an approach that enables a robot to self organize its multiple locomotion modes for accomplishing a goal-directed locomotion task. The approach taken is to focus on simple animals as a source of inspiration and to use a stochastic based control architecture known as Attractor Selection Mechanism [1016]. Despite the simplicity, it will be shown that the approach enables a robot to automatically explore different body-environment dynamics and stabilize onto particular attractors which correspond to locomotion modes relevant to the robot's goal. The robot used in this paper is a curvedbeam hopping robot, which despite its simple actuation method, possesses rich and complex dynamics depending on its mechanical configuration and environment [17-19].

The rest of the paper will be organized as follows. In the next section we will explain the basic principle of Attractor Selection Mechanism. Afterward, the Curved Beam Hopping Robot along with its simulation model will be described. In the experiment section, we will firstly explain the setup before showing the results. Finally, we will conclude the paper and suggest several possible future works.

\section{ATTRACTOR SELECTION MECHANISM}

The ability to produce non-deterministic spontaneous dynamics with emerging features has been suggested as one of the key characteristics of adaptive systems [17]. In this paper, we focus on a mathematical framework that models how stochastic perturbation, i.e. noise, can be balanced with deterministic dynamics. This framework, known as Attractor Selection Mechanism (ASM), has been used in the modeling of muscle contraction at molecular levels [10], alteration 
of gene expression depending on the environment [11], adaptive search in animals foraging [12], as well as in the development of bacteria inspired mobile robots [13-16]. To be more specific, the mathematical framework can explained by a dynamical system, represented by the Langevin equation shown in (1):

$$
\dot{s}(t)=f(s(t)) \cdot g 1(t)+\epsilon(t) \cdot g 2(t)
$$

where $s(t)$ and $f(s(t))$ represent the states and dynamics of the ASM at time $t$. Here, $\epsilon(t)$ is a noise term, while either $g 1(t)$ or $g 2(t)$ can be used to represent sensory input that indicates the suitability of the state to a given criteria and controls the behavior of ASM. As can be seen from (1), for a constant $g 2(t)$, the term $f(s(t)) \cdot g 1(t)$ becomes dominant/less-dominant in the equation when $g 1(t)$ has a high/low value such that the state transition exhibites more deterministic/stochastic dynamics respectively. Oppositely, assuming that $g 1(t)$ is constant, the term $\epsilon(t) \cdot g 2(t)$ becomes dominant/less-dominant in the equation when $g 2(t)$ has a high/low value such that the state transition behaves more stochastically/deterministically. Therefore, $g 1(t)$ should be designed such that it has a high/low value when the current state of the system is desirable/undesirable, while $g 2(t)$ should be designed oppositely.

Fig. 1 shows the basic principle of the approach proposed in this manuscript. It is shown that the interactions between the motions currently executed by the robot and the mechanical feedback from the environment, e.g. through ground contacts, may give rise to different body-environment dynamics. The question here is how to design a particular control architecture such that the self organization of the body-environment dynamics will result in the emergence of suitable locomotion modes depending on the task, without any previous knowledge about the robot's body or the environment. In our previous research, the ASM dynamics were designed with preceding knowledge about the robot's dynamics [17-18]. Here, it will be shown that an automatic exploration of different body-environment dynamics and the ability to stabilize onto particular attractors, which corresponds to distinguishable locomotion modes relevant to the task, can be enabled through a suitable control structure based on ASM. More specifically, the robot will continue to deterministically perform the current locomotion mode if it is suitable to accomplish its task, indicated by $g 1(t)$ or $g 2(t)$. Otherwise, the robot dynamics would become more random until it can find another suitable attractors.

\section{CURVED BEAM HOPPING ROBOT}

In this section, the paradigmatic robotic system, a Curved Beam Hopping Robot (CBHR), and its simulation model will be explained.

\section{A. Mechanical Structure and Model of the Robot}

Fig. 2 shows the real robot and its model in the simulation. The robot consists of three parts: a base foot, aluminum beam and a DC motor attached to a rotating mass. Therefore, the dynamics of the robot is simply driven by a single actuation

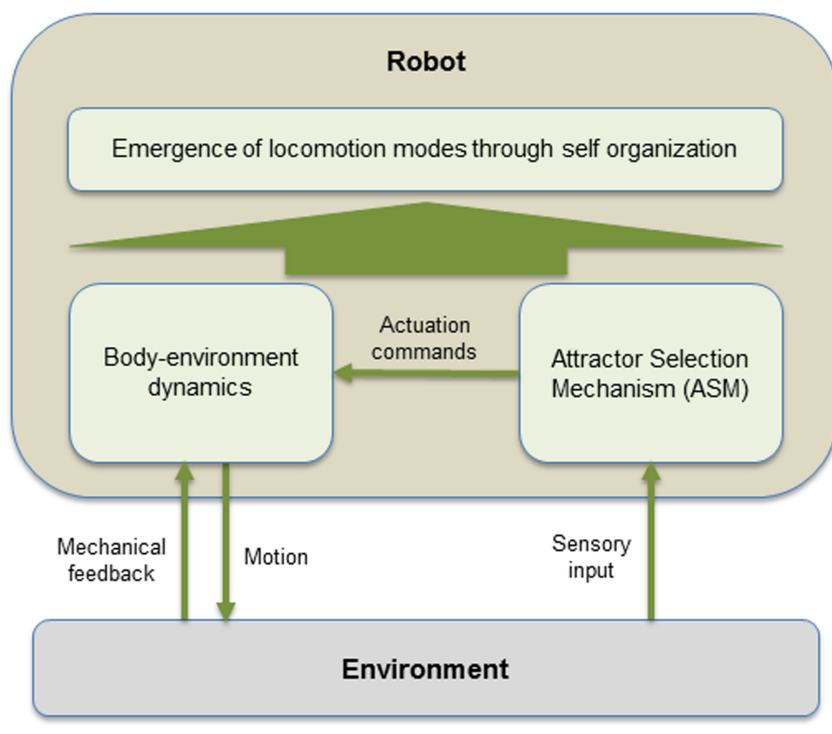

Fig. 1. The basic principle of the approach.


Fig. 2. Curved Beam Hopping Robot, the real robot (left) and the model in the simulation (right). Adjusted from [17]

frequency, i.e. the frequency of the rotating mass, and the foot's interaction with the ground.

The simulation model of the robot consists of eight joints which connects a series of rigid segments with equal lengths and motor with rotating mass as shown in Fig. 2 (right). The validity of the model as compared to the real robot has also been confirmed previously [14]. The values of the parameters used in the model can be explained as follows. Joint $J_{p i}(i=$ $1,2,3,4)$ has initial angle $\alpha_{p i}$ with stiffness coefficient $k_{p}$, while the stiffness coefficient of joint $J_{y j}(j=1,2,3,4)$ is $k_{y}$. The rotational axes of $J_{p i}$ and $J_{y i}$ are shown at the top of Fig. 2 (right). Also, $l_{y i}$ is the distance between joint $J_{p i}$ and $J_{p i+1}$ with an exception for $l_{y 4}$ which is defined as the distance between $J_{p i}$ and the point $T$. The values of the parameters described are listed down in Table I. Please note that $M$ on the table means the total mass of the robot.

\section{B. Ground Contact Model}

The ground contact model used in the simulation assumes that the ground is adequately soft, i.e. the contact points can be placed slightly below the ground, and there 
is no traction force, except the gravitational force, when the contact points are above the ground. The model has been shown to adequately describe the interaction with the environment in the real robotic system [18]. It is assumed that ground reaction force is exerted on the 4 points of the foot: $G_{p}(p=1,2,3,4)$. The reaction forces $f_{x p}, f_{y p}, f_{z p}$ is applied to those points are defined as below in the absolute Cartesian coordinate system shown in Fig. 1 (b):

$$
\begin{aligned}
f_{x p} & = \begin{cases}-k_{x}\left(x_{p}-x_{p 0}\right)-d_{x} \dot{x_{p}} & \text { if } \left.\mathrm{z}_{\mathrm{p}} \leq 0\right) \\
0 & \text { if } \left.\mathrm{z}_{\mathrm{p}}>0\right)\end{cases} \\
f_{y p} & = \begin{cases}-k_{y}\left(y_{p}-y_{p 0}\right)-d_{y} \dot{y_{p}} & \text { (if } \left.\mathrm{z}_{\mathrm{p}} \leq 0\right) \\
0 & \text { if } \left.\mathrm{z}_{\mathrm{p}}>0\right)\end{cases} \\
f_{z p} & = \begin{cases}f_{c}-f_{g} & \left(\text { if } \mathrm{z}_{\mathrm{p}} \leq 0\right) \\
-f_{g} & \left(\text { if } \mathrm{z}_{\mathrm{p}}>0\right)\end{cases} \\
f_{c} & = \begin{cases}-k_{z} z_{p}-d_{z} \dot{z_{p}} & \text { (if } \left.\dot{\mathrm{p}_{\mathrm{p}}} \leq 0\right) \\
0 & \text { (if } \left.\dot{z_{\mathrm{p}}}>0\right)\end{cases}
\end{aligned}
$$

where $k_{x}, k_{y}, k_{z}$ are the stiffnesses along with $x, y, z$-axis and $d_{x}, d_{y}, d_{z}$ are the damping coefficients. $\left(x_{p}, y_{p}, z_{p}\right)$ is the current position of the point $G_{p}$, while $x_{p 0}, y_{p 0}$ represent the coordinates of the point in each axis at touchdown. $f_{g}$ is gravitational force. Here, $f_{c}$ doesn't exert on the points when $\dot{z}_{p}>0$ for the purpose of removing the pulling-down effect of the force, as suggested in [20]. The values of the parameters are defined in Table 1.

\section{Dynamics of the Robot}

As explained by the concept shown in Fig. 1, the dynamics of the robot depends on the interactions among ASM, the robot's mechanical body and the environment. To demonstrate the concept, we change the parameters values which corresponds to different mechanical configuration of the the robot's body. Throughout this paper, each set of these parameter values will simply be referred to as one mechanical configuration (mech. conf.). Here, we use three different mechanical configurations by varying the angle between the beam and the foot, and the stiffness of the beam. The parameter values can therefore be described as follows: $k_{p 2} \sim k_{p 4}=13, \theta_{b}=0 ; k_{p 2} \sim k_{p 4}=13, \theta_{b}=-\pi / 6$; $k_{p 2} \sim k_{p 4}=7, \theta_{b}=0$.

Fig. 3 (a) and (b) shows the trajectory of the robot at two fixed actuation frequencies, i.e. 25 and $35 \mathrm{rad} / \mathrm{s}$, for different mechanical configurations. Each trajectory represents the position of the center of the foot in a ten second simulation. In

\begin{tabular}{|c|c|c|c|c|c|}
\hline Name & Value & Name & Value & Name & Value \\
\hline$l_{o}$ & $0.160 \mathrm{~m}$ & $l_{y 2}$ & $0.141 \mathrm{~m}$ & $\alpha_{p 4}$ & $1.803 \mathrm{rad}$ \\
\hline$l_{r}$ & $0.030 \mathrm{~m}$ & $l_{y 3}$ & $0.135 \mathrm{~m}$ & $k_{p 1}$ & $15 \mathrm{~N} / \mathrm{rad}$ \\
\hline$l_{b 1}$ & $0.305 \mathrm{~m}$ & $l_{y 4}$ & $0.145 \mathrm{~m}$ & $k_{p 2} \sim k_{p 4}$ & $13 \mathrm{~N} / \mathrm{rad}$ \\
\hline$l_{b 2}$ & $0.275 \mathrm{~m}$ & $\alpha_{p 1}$ & $2.554 \mathrm{rad}$ & $k_{y 1} \sim k_{y 4}$ & $3.517 \mathrm{~N} / \mathrm{rad}$ \\
\hline$l_{b 3}$ & $0.082 \mathrm{~m}$ & $\alpha_{p 2}$ & $2.570 \mathrm{rad}$ & $m$ & $0.030 \mathrm{~kg}$ \\
\hline$l_{y 1}$ & $0.151 \mathrm{~m}$ & $\alpha_{p 3}$ & $1.665 \mathrm{rad}$ & $M$ & $0.331 \mathrm{~kg}$ \\
\hline \hline$k_{x}$ & $5 \mathrm{~N} / \mathrm{m}$ & $k_{y}$ & $5 \mathrm{~N} / \mathrm{m}$ & $k_{z}$ & $10^{5} \mathrm{~N} / \mathrm{m}$ \\
\hline$d_{x}$ & $5 \mathrm{Ns} / \mathrm{m}$ & $d_{y}$ & $5 \mathrm{Ns} / \mathrm{m}$ & $d_{z}$ & $10 \mathrm{Ns} / \mathrm{m}$ \\
\hline
\end{tabular}

TABLE I

THE VALUES OF THE PHYSICAL PARAMETERS

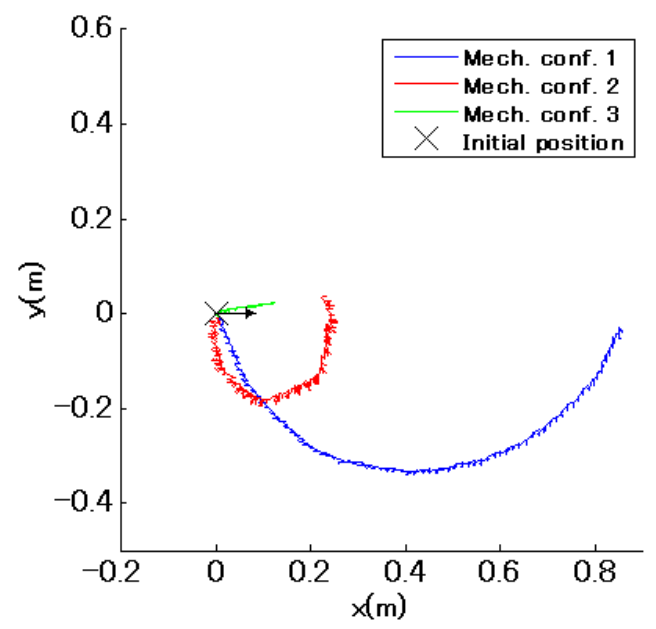

a) $\omega=25$

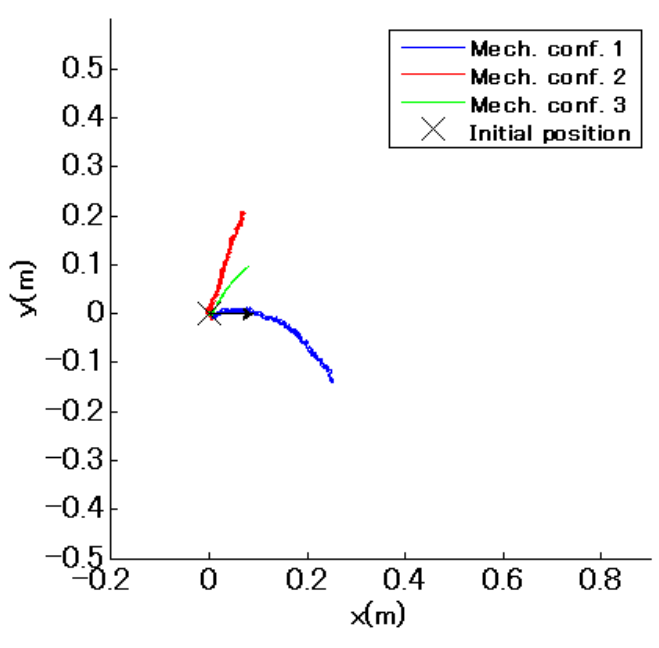

b) $\omega=35$

Fig. 3. A variety of robot dynamics due to different mechanical configurations despite having the same actuation frequency of $\omega=25,35$.

this paper, it will be shown that without any knowledge about its mechanical configurations, the robot will be able to self organize its body-environment dynamics in order to perform goal-directed locomotion by exploring and stabilizing onto suitable attractors.

\section{IMPLEMENTATION OF THE ATTRACTOR SELECTION MECHANISM}

In order to enable the robot to perform goal-directed locomotion, we implement the basic equation of Attractor Selection Mechanism explained in (1). Here, we choose $g 1(t)=1$, and $g 2(t)=g(t)$, and use the following equations.

$$
\begin{aligned}
\dot{\omega} & =f(\omega)+\epsilon \cdot g(t) \\
f(\omega) & = \begin{cases}\left\{\exp \left(-\omega+\omega_{b}\right)-1\right\} p & \text { if } \omega \leq \omega_{b} \\
0 & \text { if } \omega_{b}<\omega<\omega_{t}(7) \\
-\left\{\exp \left(\omega-\omega_{t}\right)-1\right\} p & \text { if } \omega \geq \omega_{t}\end{cases}
\end{aligned}
$$



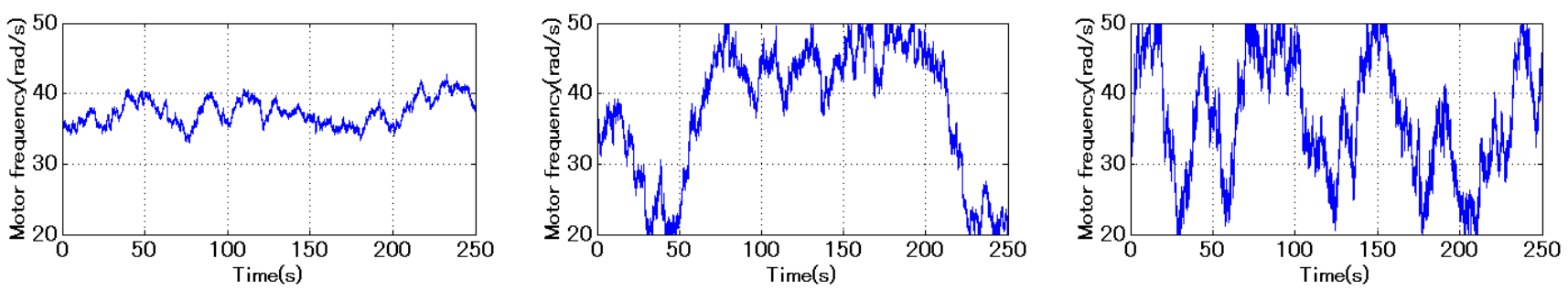

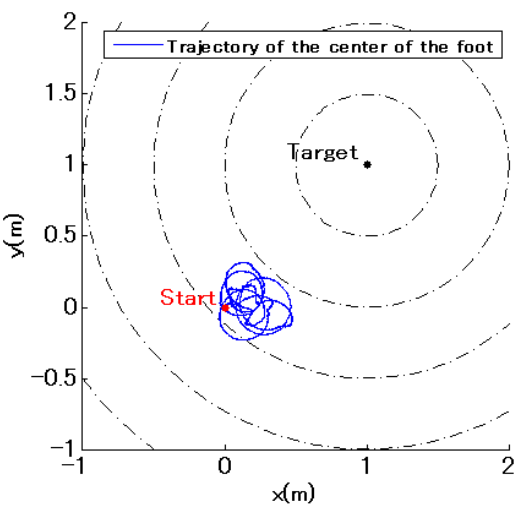

a) Noise level $=50$

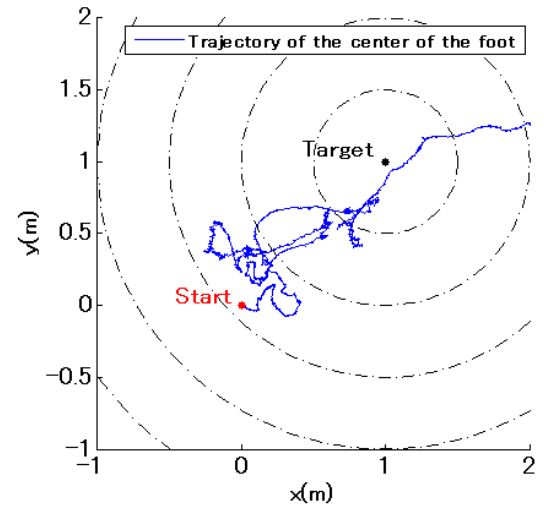

b) Noise level $=150$

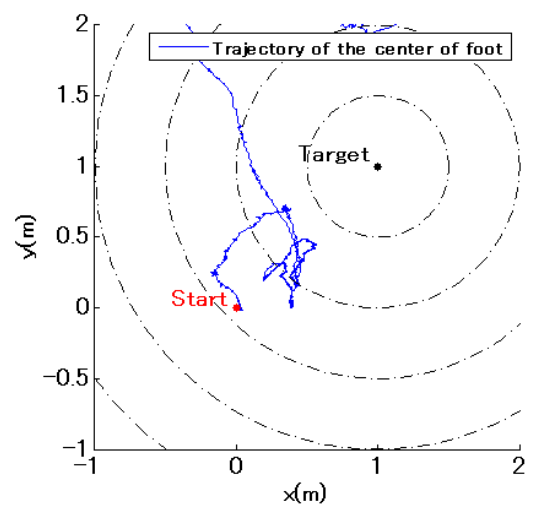

c) Noise level $=250$

Fig. 4. The time series of the motor frequency (top) and the robot trajectories from top-view (bottom) for different levels of noise, mech. conf. 1.

$$
g(t)= \begin{cases}\exp [\{d(t)-d(t-1)\} q] \\ g_{b} & \text { if } g(t)<g_{b} \\ g_{t} & \text { if } g(t)>g_{t} \\ 1 & \text { if }|d(t)-d(t-1)|>\Delta d_{m}\end{cases}
$$

where $\omega$ represents the motor frequency, while $\epsilon$ represents internally generated artificial noise with Gaussian distribution. As the robot barely moves for motor frequency below $20 \mathrm{rad} / \mathrm{s}$, the frequency range is limited in a range of $20 \sim$ $50 \mathrm{rad} / \mathrm{s}$ through $f(\omega)$, where $\omega_{b}, \omega_{t}$ are the bottom and top frequency respectively. In between the two frequencies, the change of $\omega$ over time will be driven by $\epsilon$ to enable the exploration of different body-environment dynamics. In order to let the robot perform goal-directed locomotion, $g(t)$ is set to be a function of the difference between $d(t)$ and $d(t-1)$, where $d(t)$ is distance at the time $t$. As can be seen from (7), $g(t)$ adjusts the size level of $\epsilon$ such that the robot's tendency to stochastically search for a more suitable dynamics is decided by whether it is approaching the goal or not. Also, in order to keep a moderate level of stochasticity, $g(t)$ is saturated with bottom value $g_{b}$ and top value $g_{t}$. The last condition, $g(t)=1$ when $|d(t)-d(t-1)|>\Delta d_{m}$, is implemented to avoid small oscillations of the robot when its foot are under the ground due the used ground contact model. $p, q$ are adjustable coefficients. All the used parameter values are shown in table 2 . It is assumed that based on the aforementioned equations, through the dynamics of $\omega$, the robot will be able to explore and stabilize itself onto useful distinguishable locomotion modes depending on whether it is approaching its goal or not.

\section{SIMULATION EXPERIMENT}

Having described the implementation detail of the ASM, the section will describe the performed simulation experiment based on the model shown in Fig. 2 (right). The goal of the experiment is to confirm whether the approach will enable the robot to perform goal-directed locomotion by exploring different body-environment dynamics and stabilizing itself onto suitable attractors which correspond to distinguishable locomotion modes relevant to the task.

\section{A. The Effect of The Noise Level}

As the approach is supposed to enable the robot to explore a variety of body-environment dynamics, the first set of experiments aims to observe the effect of different levels of noise to the robot's behavior explained in (6). Fig. 4 shows the examples of trajectories and how the motor frequency changes in 250 second simulation for different level of the internally generated artificial noise $\epsilon(t)\left(\mathrm{rad} / s^{2}\right)$ in (6), defined by the standard deviation. Fig. 4 (a), (b) and (c) show examples for standard deviation 50,150 and 250, respectively. Here, the used mechanical configuration is the first one, i.e. mech. conf. 1 described in the previous section. The robot starts from initial position $(0,0)$, while

\begin{tabular}{|c|c|c|c|}
\hline Name & Value & Name & Value \\
\hline$\omega_{b}$ & $20 \mathrm{rad} / \mathrm{s}$ & $p$ & $10^{4}$ \\
$\omega_{t}$ & $50 \mathrm{rad} / \mathrm{s}$ & $q$ & $10^{6}$ \\
$g_{b}$ & 0.01 & $\Delta d_{m}$ & $0.005 \mathrm{~m}$ \\
$g_{t}$ & 2 & & \\
\hline
\end{tabular}

TABLE II

THE VALUES OF THE ASM PARAMETERS 


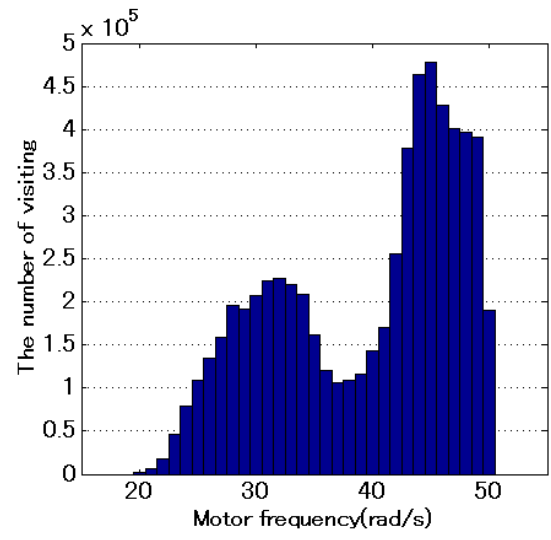

a) Mech. conf. 1

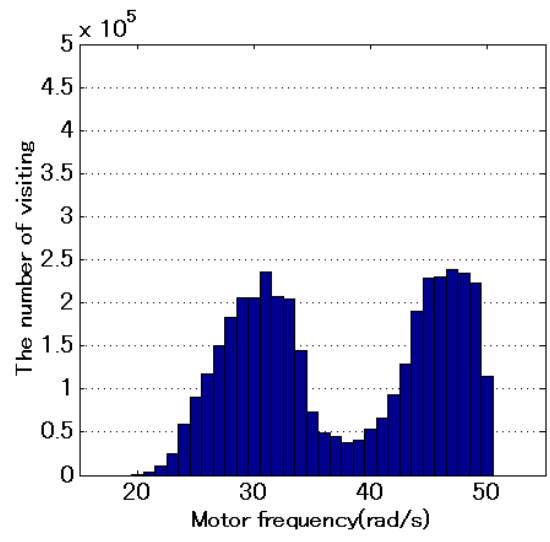

b) Mech. conf. 2



c) Mech. conf. 3

Fig. 6. The histogram of the visited frequencies for different mechanical configurations showing the emergence of different locomotion modes for different mechanical configurations.

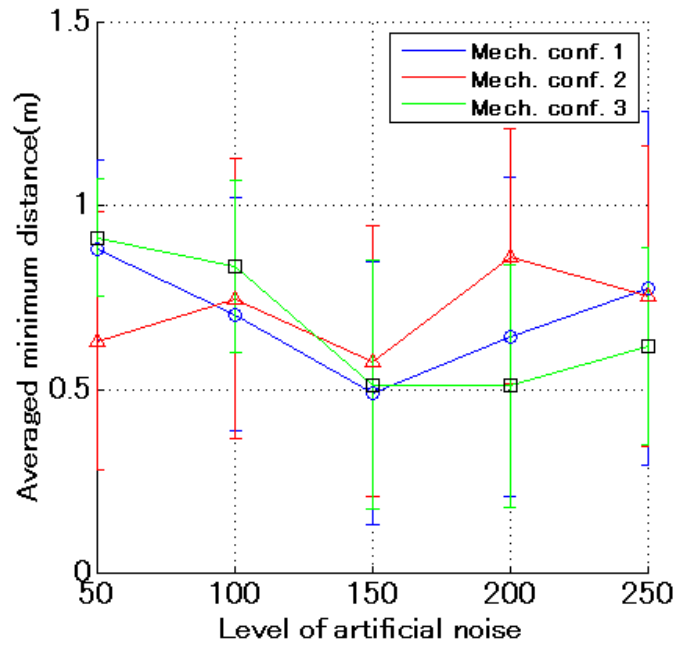

Fig. 5. The goal-directed locomotion performance, i.e. the average of the minimum distance to the goal, for various mechanical configurations based on the different levels of noise $\epsilon(t)$.

its goal is placed at $(1,1)$. As can be seen, a suitable size of noise level let the robot adequately explore different body-environment dynamics which enables itself to perform goal-directed locomotion (Fig.4 (b)). If the noise level is too low, the robot will not be able to adequately explore its body-environment dynamics (Fig. 4 (a)). On the other hand, a too high noise level makes it difficult for the robot to stabilize itself onto particularly useful attractors, which results in a failure in accomplishing the task of reaching the goal. Having confirmed the ability of the robot to explore and stabilize itself onto different body-environment dynamics for one mechanical configuration, we perform the same set of experiments for mech.conf. 2 and mech. conf. 3 and confirm similar behaviors. Fig. 5 also shows the minimum distance of the robot from the target position, averaged from ten trials along with its standard deviation. It is however interesting to notice that the best level of noise can be different according to mechanical configurations: 150 for mech. conf. 1 and 2; 200 for mech. conf. 3. How different mechanical configurations lead to different dynamics will be explained further in the next section.

\section{B. The Emerging Locomotion Modes}

In order to understand the behavior of the robot for different mechanical configuration, we plot the histogram of the visited frequencies for each configuration shown in Fig. 6 . The figure is obtained by observing the motor frequency for each sampling period in the simulations, which were selected from the ones with the best level of noise suggested in Fig. 5. It must also be noticed that there are two phases in the dynamics of the robot: the flight phase, where the robot goes through a transition from moving along the ground to hopping in the air, and the stance phase, which begins with the strike of the robot's foot on the ground. The frequencies used in Fig. 6 were only collected during flight phases as the robot only does minor oscillations moving during stance phases. For different mech. conf., the total time periods spent for flight and stance phase through the ten trials at each best noise level can be seen in Table 3. From Fig. 6, it can also be seen that the most frequently visited frequencies for mech. conf. 1 are approximately $32 \mathrm{rad} / \mathrm{s}$ and $45 \mathrm{rad} / \mathrm{s}$. For mech. conf. 2, the most visited frequencies are approximately the 31 and $47 \mathrm{rad} / \mathrm{s}$, while for mech. conf. 3 , they are approximately 21 and $49 \mathrm{rad} / \mathrm{s}$.

Fig. 7 shows ten second trajectories of the robot at the aforementioned attractors, i.e. the most visited frequencies, for each mechanical configurations. It is interesting to notice, that while the trajectories are not exactly the same for each configuration, they have opposite moving direction. It also indicates why they are chosen as attractors which correspond to

\begin{tabular}{|l||c|c|c|}
\hline & Flight phase & Stance phase & Total period \\
\hline Mech. conf. 1 & $633.2 \mathrm{~s}$ & $1866.8 \mathrm{~s}$ & $2500.0 \mathrm{~s}$ \\
Mech. conf. 2 & $388.4 \mathrm{~s}$ & $2111.6 \mathrm{~s}$ & $2500.0 \mathrm{~s}$ \\
Mech. conf. 3 & $130.3 \mathrm{~s}$ & $2369.7 \mathrm{~s}$ & $2500.0 \mathrm{~s}$ \\
\hline
\end{tabular}

TABLE III

LOCOMOTION PERIODS IN DIFFERENT PHASES FOR ALL THE TRIALS 


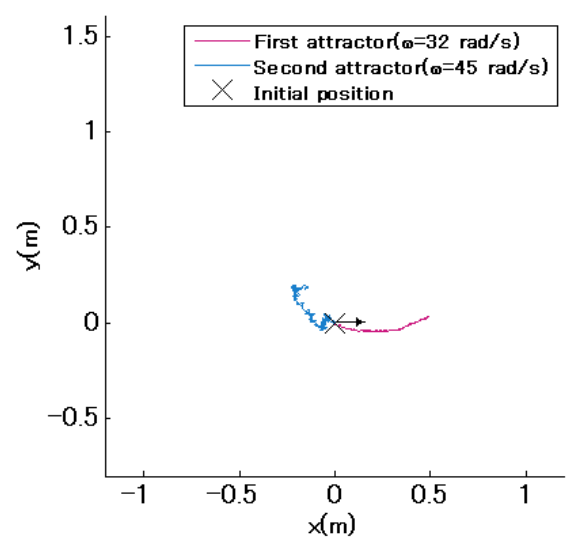

a) Mech. conf. 1



b) Mech. conf. 2

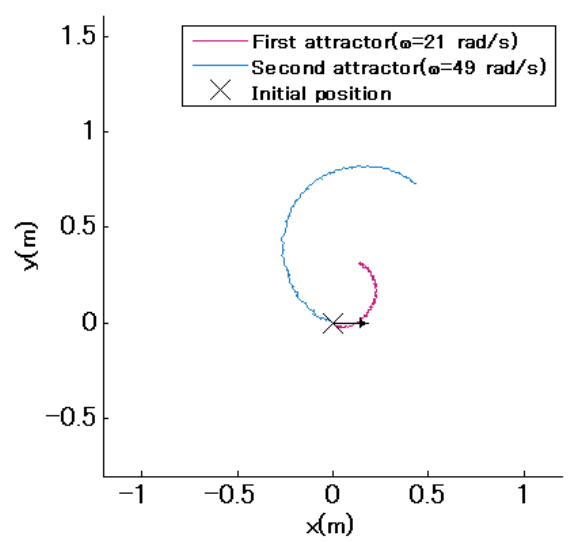

c) Mech. conf. 3

Fig. 7. The trajectories of the center of the foot at attractors suggested by Fig. 6 which corresponds to locomotion modes favorable for each mechanical configuration.

distinguishable and favorable locomotion modes discovered by the robot in each mechanical configuration to accomplish the goal-directed locomotion task.

\section{CONCLUSIONS}

This paper demonstrates the realization and utilization of multimodal locomotion that enable robots to accomplish a goal-directed locomotion task. The approach taken is based on the self organization of the robot's body-environment dynamics through a simple control structure known as Attractor Selection Mechanism (ASM). Despite its simplicity, ASM enables the robot to explore its body-environment dynamics by using internally generated noise and stabilize itself onto particular attractors corresponding to locomotion modes favorable for the task. The result has been confirmed for different mechanical configurations of a Curved Beam Hopping Robot, which possesses rich and complex body-environment dynamics in spite of its simple actuation method.

For future works, it is interesting to further understand the relationship between the parameter values in the ASM and the mechanical configurations. We also plan to extend the experiment with our real Curved Beam Hopping Robot.

\section{ACKNOWLEDGMENT}

This study was supported by the Swiss National Science Foundation Grant No. PP00P2123387/1 and the Swiss National Science Foundation through the National Centre of Competence in Research Robotics. The first author was also supported by Tokyo Institute of Technology - ETH Zürich Student Exchange Program.

\section{REFERENCES}

[1] Auke J I, Crespi A, Ryczko D and Cabelguen J M, "From swimming to walking with a salamander robot driven by a spinal cord model," Science, 2007, 515: 1416-1420.

[2] Emerson S B, "The interaction of behavioral and morphological change in the evolution of a novel locomotor mode: 'flying' frogs," Evolution, 1990, 44: 1931-46.
[3] Hosoda K, Takuma T, Nakamoto A and Hayashi S, "Biped robot design powered by antagonistic pneumatic actuators for multi-modal locomotion," Robot. Auton. Syst., 2008, 56: 46-53.

[4] Pfeifer R, Lungarella M and Iida F, "Self organization, embodiment and biologically inspired robotics," Science, 2007, 318: 1088-1093.

[5] Iida F and Preifer R, "Sensing through mechanical dynamics," Robotics and Autonomous Systems, 2006, 54: 631-640.

[6] Nurzaman SG, Culha U, Brodbeck L, Wang L and Iida F, "Active sensing with in-situ adjustable sensor morphology," PLoS ONE, 2013, 8(12): e84090.

[7] Shim Y and Husbands P, "Chaotic exploration and learning of locomotion behaviors," Neural Comput., 2012, 24: 21852222.

[8] Berg H C, "Motile behavior of bacteria,", Phys. Today, 2000, 53: 249.

[9] Pierce-Shimomura J T, Morse T M and Lockery S R, "The fundamental role of pirouettes in Caenorhabditis elegans Chemotaxis," $J$. Neurosci., 1999, 19: 955769.

[10] Yanagida T, Ueda M, Murata T, Esaki S and Ishii Y, "Brownian motion, fluctuation and life", BioSystems, 2007, 88: 22842.

[11] Kashiwagi A, Ureba I, Kaneko K and Yomo T, "Adaptive response of a gene network to environmental changes by fitness-induced attractor selection," PLOS ONE, 2006, 1(1): e49.

[12] Kashiwagi A, Ureba I, Kaneko K and Yomo T, "From Levy to Brownian: A computational model based on biological fluctuation," PLoS ONE, 2011, 6(2): e16168.

[13] Nurzaman S G, Matsumoto Y, Nakamura Y, Koizumi S and Ishiguro $\mathrm{H}$, "Attractor selection based biologically inspired navigation system," Proc. of the 39th Int. Symp. On Robotics, 2008, pp 83742.

[14] Nurzaman S G, Matsumoto Y, Nakamura Y, Shirai K, Koizumi $\mathrm{S}$ and Ishiguro $\mathrm{H}$, "An adaptive switching behavior between Levy and Brownian random search in a mobile robot based on biological fluctuation," Proc. of the IEEE Int. Conf. on Intelligent Robots and Systems (IROS), 2010, pp 1927-1934.

[15] Nurzaman SG, Matsumoto Y, Nakamura Y, Shirai K, Koizumi S, Ishiguro $\mathrm{H}$, "Bacteria inspired underactuated mobile robot based on a biological fluctuation," Adaptive Behavior, 2012, 20(4): 225-236.

[16] Nurzaman SG, Matsumoto Y, Nakamura Y, Koizumi S, Ishiguro $\mathrm{H}$, "'Yuragi' based adaptive mobile robot search with and without gradient sensing: From bacterial chemotaxis to Levy walk," Advanced Robotics, 2011, 25(16): 2019-2037.

[17] Nurzaman SG, Yu X, Kim Y and Iida F, "Guided Self-Organization in a Dynamic Embodied System Based on Attractor Selection Mechanism," Entropy, 2014, 16: 2592-2610.

[18] Nurzaman SG, Yu X, Kim Y and Iida F, "Goal-directed multimodal locomotion through coupling between mechanical and attractor selection dynamics," Bioinspiration \& Biomimetics, In press.

[19] Reis M and Iida F, "An Energy-Efficient Hopping Robot Based on Free Vibration fo a Curved Beam," IEEE/ASME Trans. Mechatronics, 2014, 19: 300-311.

[20] Brubaker M A, Sigal L and Fleet D J, "Estimating contact dynamics," Proc. of the IEEE Int. Conf. on Computer Vision, 2009, pp 2389-2396. 